\title{
Research on the Protection of Historical and Cultural Blocks
}

\author{
Ruxu Zhang \\ Nanyang Institute of Technology, Nanyang, Henan, 473004
}

Keywords: Historical and Cultural Blocks, Protection Method, Historical Value

\begin{abstract}
With the acceleration of urbanization process, many countries and regions in the growing urban construction efforts, mainly in order to be able to continue to move into, increase the population, to provide adequate and pleasant living environment. Historical and cultural blocks reflect the history of the city, culture, can show the city's historical style, so in the process of rapid urbanization is facing protection problems. However, in the past China's urban history and cultural street reconstruction activities, the reason for the development and constructive damage, because of the lack of historical and cultural blocks of the correct understanding of the value, and in pursuit of economic interests ignored the city's historical context Of the continuation, coupled with some places in the protection of the concept of planning there is a deviation, so there has been the loss of local characteristics, behind closed doors, from the actual, poor implementation and other issues. In addition, in 2014 China has just promulgated a new urbanization planning, more emphasis on cultural heritage, highlighting the city's characteristics and personality. In this case, experts in various fields of society began to advocate the rational protection of historical and cultural neighborhoods in order to preserve more and more precious historical culture.
\end{abstract}

\section{Introduction}

In recent years, the concept of public participation in some of the relevant concepts in the protection of historical and cultural blocks in China was introduced, because of the Western countries to participate in the planning of public influence. However, although the introduction of public participation but less effective. At present, the planning mode of the historical and cultural blocks is still a top-down "elite" model with the government and experts as the main body. The protection planning is often separated from society, lack of maneuverability and extensive supervision, lack of care for the residents And people-oriented concept, to absorb the local people willing to carry out the transformation of the small, can not reflect the people's urgent desire, it is difficult to mobilize the enthusiasm of residents to actively participate. However, the public, especially those living in the historical and cultural blocks of the residents, it is difficult to really participate in the protection of historical and cultural blocks in the planning process, this situation is China's historical and cultural district protection planning work unfavorable factors, because it makes the public on the protection planning Lack of a comprehensive understanding, and thus make the protection of planning work is full of difficulty. At the same time, the public awareness of democracy continues to increase, more and more people began to pay attention to their own things around, and eager to participate in the social and public affairs. Therefore, to increase the protection of historical and cultural blocks of social basis, has gradually become China's historical and cultural blocks of the important part of the planning work, the introduction of public participation as an objective need.

\section{Research on the Protection Theory of Historical and Cultural Blocks}

China's history and cultural neighborhoods started relatively late. In 1982, the State Council promulgated the first batch of national historical and cultural city, and in the "State Council approved the State Construction Committee and other departments on the protection of China's 
historical and cultural city of the notice of the notice" for the first time referred to "to reflect the history and culture of the old city , The site, cultural relics ... ... to take effective measures, strict protection, ... ... in these monuments around a certain range of protected areas, within the scope of the new, expansion, alteration project should take the necessary restrictions. It can be seen that the concept of historical district has not yet formed, but has recognized the protection of a certain extent outside the monuments.

By 2008, the concept of historical and cultural blocks has been further clarified. The State Council promulgated the "historical and cultural city name town name village protection regulations" provides that the historical and cultural blocks refers to the preservation of cultural relics are particularly rich, historical buildings focus on the film, to a more comprehensive and true reflection of the traditional pattern, historical style, and a certain scale area.

2005 historical and cultural city protection planning norms put forward the historical and cultural blocks should have a more complete style, constitute the historical style of historical buildings and historical environmental elements is basically the history of retention of the original, historical buildings shall not be removed, the historical environment Elements of the repair and maintenance, neighborhood streets should maintain the original historical pattern and spatial scale. To the 2008 promulgated the "historical and cultural city name town village protection regulations", proposed to protect the district pattern and style, and historical and cultural closely related to the natural landscape, water, ancient trees, etc., neighborhood protection more emphasis on the protection of historical reality Sex, style integrity and as far as possible to maintain the function of the continuation of the district, we can see "historical and cultural city name town village protection regulations" pay more attention to the integrity of the district pattern style, the protection of more content, more scientific and reasonable expression. From the "preservation of the appearance of historical buildings, improve the living conditions of the room to improve the urban infrastructure" can be seen not only focus on the protection of the building, pay more attention to the protection of the appearance of historical buildings, while the transformation of internal facilities also reflects the people-oriented thinking, Of the living environment to improve the conditions to be taken seriously.

To the 2012 issue of "historical and cultural city name town village protection planning requirements (Trial)" on the historical and cultural blocks of the protection of the planning content has been deepened and refined. It is pointed out that the protection plan of historical and cultural neighborhoods should include the assessment of historical and cultural values, characteristics and existing problems, to determine the protection principles, content and delineation of protection, and to formulate appropriate protection and control measures. Further clarify the protection of content and scope, highlighting the value of the neighborhood characteristics. At the same time, it is proposed to classify and protect the buildings and structures within the protection area of the block, including cultural relics protection units, historical buildings, traditional buildings and other buildings, and classify the design requirements such as building height, volume color, appearance image and color, This classification of the traditional style of architecture from the 2005 "norms" in the "general building" in the separation, emphasizing the protection of traditional style buildings, and proposed to keep the vitality of the block, the continuation of traditional culture planning measures to improve traffic , Infrastructure, public service facilities, living environment and so on.

\section{Determine the Principle of Protection Zoning}

The demarcation of the core protected areas should be based on the principle of "complete spatial structure, traditional style, continuous visual landscape" [20], cultural and historical monuments, historical buildings, and the outer boundary lines of traditional streets along the historical and cultural streets The construction of the control area should be based on the "continuation of style, asymptotic over the" principle, within a certain distance outside the core protected areas, combined with streets, rivers, walls, Mountain and other obvious geographical boundaries to delineate. [19] Protection of neighborhoods within the cultural relics, historical buildings, fish bones pattern road network, continuous, varied streets and alleys, traditional commercial living space of the overall environmental atmosphere, protected with near, modern 
events, celebrity activities Related historical sites, celebrity residence, historical streets and other historical and cultural resources, the overall continuation of the city history and culture context, spatial structure.

Rational use, and take into account the development. Some of the architectural style is not obvious, poor quality of the building is divided into construction control zone, in the construction of control zones to carry out appropriate construction control to protect the integrity of the core protected area. Improve the function and layout, optimize the regional environmental quality and spatial landscape, update the facilities, change the historical building of the negative protection of the state, so that the historical buildings and their environment both to maintain the characteristics and meet the requirements of modern development, improve the use of historical buildings, The Development Model of the Protection of Cultural Blocks and the Favorable Circulation of Regional Development. So that the historical and cultural blocks and modern urban development harmonious coexistence, mutual promotion.

The scope of the protection zone should be scientific and effective, must be combined with zoning implementation considerations, operational performance in the following areas: First, strengthen communication with local governments and relevant departments, coordination with the relevant planning, and urban development. Second, to ensure the viability of management, as far as possible to roads, rivers, buildings and other easy to identify the reference for the sector, and some sites buried in the ground, the surface without reference points, but also take a few special points to form a multilateral line , Zoning and administrative divisions in coordination, to avoid the "four to" and "cross" and other difficult to implement control of the zoning. Third, the protection of zoning should be able to effectively protect the historical and cultural heritage, but also conducive to protecting the lives of residents, the protection of people's livelihood is to protect the heritage area of the intangible cultural heritage. Many cities in our country have established a special demonstration system for the protection of the plan, the more successful is Shanghai, through the adoption of the proceedings and written advice to conduct a special argument, coordination of the implementation of the implementation of the protection plan implementation of contradictions, protection of protection planning Practical operability.

First of all, the scope of the protection should be to protect the historical and cultural blocks of the authenticity and integrity requirements, a clear historical and cultural blocks of the value of the composition of the historical and cultural blocks and the surrounding environment as a whole to ensure that the historical and cultural blocks of the core protection of the real Sex. Construction of control zones should respect history and based on the status quo, to protect the historical and cultural blocks of the historical style of integrity, and coordination of urban development and historical and cultural blocks of the contradiction between the protection of land.

Secondly, in the City historical and cultural district protection zone planning timing, should consider the delimitation of the boundaries of the operability, and historical and cultural blocks in the administrative feasibility of the Department can not ignore the historical information, the scope of the protection of zoning as " Four to "the ratio of amplification. Only clear the boundaries of historical and cultural blocks in order to develop reasonable and specific provisions for the historical and cultural blocks of daily management and planning control to provide the basis. The scope of the protection zone should be scientific and effective, must be combined with zoning implementation considerations, operational performance in the following areas: First, strengthen communication with local governments and relevant departments, coordination with the relevant planning, and urban development. Second, to ensure the viability of management, as far as possible to roads, rivers, buildings and other easy to identify the reference for the sector, and some sites buried in the ground, the surface without reference points, but also take a few special points to form a multilateral line , Zoning and administrative divisions in coordination, to avoid the "four to" and "cross" and other difficult to implement control of the zoning. Third, the protection of zoning should be able to effectively protect the historical and cultural heritage, but also conducive to protecting the lives of residents, the protection of people's livelihood is to protect the heritage area of the intangible cultural heritage. 
Again, the historical and cultural blocks are not immutable, and it itself is a dynamic process of change. The protection zoning should be dynamically adjusted according to the actual situation of the protection of historical and cultural blocks and the actual situation of urban development. Such as the impact of cultural relics protection units around the surrounding style of the building, due to property rights and other circumstances can not be immediately demolition, you can recently put forward their own control requirements, long-term combination of urban development trends to a better direction. Finally, the protection of regionalization should reflect a certain degree of dynamic, at present, is the preparation of historical and cultural city protection planning, the historical and cultural blocks of the protection of the delineation of the historical and cultural blocks will have an impact on the protection.

\section{Conclusion}

Historic district is an integral part of the city's body, since it is the organism will be endless metabolism. Historic blocks are not solidified historical specimens, can not use the "museum" ideas to protect and build historical blocks. The architectural features of the neighborhood, the street pattern, the city style and so on, if not with the people of this relationship, they and the museum in the dusk of the deadly distinction between what? A difficult to implement the harsh protection plan can not really protect the historical and cultural heritage you want to protect. The main purpose of the protection of historical and cultural blocks is to cultivate a living context! This requires the active participation of the various communities. Not for the rigid material carrier, deliberately shield people on the historical history of the old things reasonable use and update. In a real historical and cultural value of the environment to enjoy a cup of tea, sip a pot of wine, taste a meal or perhaps than a virtual "window" on the history and culture of the ceremony more appreciate what is the essence of history and culture. Participatory activities are really the best way to keep history and culture into life and to extend it. Historic neighborhoods are undoubtedly the best place to go. Dialectical view of the so-called historical district of the original, with the development of the eyes of the history of the future view of the relationship between people and things, insight into the evolution of the trend of the district, in the development of dynamic protection, reasonable update, in order to truly maximize the historical and cultural heritage and Its heritage to the future.

\section{References}

[1] Qian Qian, Zhen Feng, Wang Bo, Xiong Lifang. Study on the Method and Method of Compiling Urban Industrial Planning Based on Public Participation [J].Urban Development Studies 2012 (11)

[2] Xiong Xixian. Public participation in the protection planning of historical and cultural blocks [J]. Jiangsu Urban Planning. 2012 (09)

[3] Zhuang Xiaoping, Zhu Hong, Deng Suqiu. Study on Institutional Ethics of Comparative Study on Resident Tourism Perception [J]. Journal of Tourism 2012 (03)

[4] Wang Lei, Zhang Yao. Study on the Cognition and Willingness of Villagers' Participation in Rural Planning in Southern Jiangsu Province - A Case Study of Changshu City, Jiangsu Province [J]. Urban Planning. 2012 (02)

[5] Xu Mingyao, Tao Dekai. Exploration and Reflection on Public Participation in Urban Planning in the New Period - Taking Nanjing Urban Master Plan as an Example [J]. Urban Planning. 2012 (02)

[6] Zhang Cheng, Ye Bin, Su Ling. The overall view of historical and cultural city protection in the transition period - Taking Nanjing as an example [J]. Urban Planning. 2011 (S1) 